erwähnte Kontinuum des Sonnenlichtes zwischen 910 und etwa $800 \AA$. Hier hat der Ionisierungsquerschnitt eine starke Struktur durch eine Reihe von $\mathrm{Au}$ toionisationsvorgängen. Der gemessene Wirkungsquerschnitt für die Autoionisation im Bereich des ${ }^{4} \mathrm{~S}^{0}$-Kontinuums kann den 10 fachen Wert des ungestörten Ionisationskontinuums annehmen, wie der Meßpunkt für die Wellenlänge $747 \AA$ zeigt. Es steht aber nicht fest, ob der aufgenommene Meßwert dem Maximum der autoionisierenden Termgruppe entspricht. Es ist sogar anzunehmen, daß die Absorption im Bereich der autoionisierenden Terme wesentlich höher ist. Selbst wenn die Koinzidenz eines Sauerstoffterms und einer der in dem Linienpaket bei $747 \AA$ enthaltenen Linien exakt sein sollte, so ist ihre Intensität nur ein Bruchteil der für die Berechnung des Wirkungsquerschnittes in Rechnung gesetzten Intensität der ganzen Liniengruppe. Unterhalb $800 \AA$ sind die autoionisierenden Terme des Sauerstoffatoms von geringerer Bedeutung für die Sauerstoffionisation durch die Strahlung der Sonne, da in diesem Wellenlängenbereich nur eine geringe Koinzidenz zwischen den Emissionslinien des Sonnenspektrums und den autoionisierenden Termen des Sauerstoffs besteht. Unter Umständen ist eine solche Koinzidenz in der Nähe von $770 \AA$ vorhanden. Mit Hilfe der Intensitätsangabe des Sonnenspektrums und des gemessenen Wirkungsquerschnittes des Sauerstoffatoms läßt sich eine Abschätzung des Ausmaßes der Sauerstoffionisierung durchführen. Dieses soll jedoch im Rahmen der vorliegenden Arbeit nicht geschehen und auf einen späteren Zeitpunkt zurückgestellt werden, bis die geplante Vermessung des Ionisierungsquerschnittes mit dem Hintergrund eines Kontinuums erfolgt ist. Eine solche Messung wird eine genauere Abschätzung über den Beitrag der autoionisierenden Terme zur Ionisierung des Sauerstoffatoms liefern.

Wir danken der Deutschen Forschungsgemeinschaft für ihre Unterstützung dieser Arbeit.

\title{
Photoionisationsuntersuchungen an Atomstrahlen
}

III. Der Ionisierungsquerschnitt des atomaren Stickstoffs

$$
\text { F. J. Comes und A. Elzer }
$$

Institut für Physikalische Chemie der Universität Bonn

(Z. Naturforsch. 23 a, 133-136 [1968] ; eingegangen am 23. September 1967)

\begin{abstract}
The photoionization cross section of atomic nitrogen has been measured between the ionization limit and $432 \AA$. The experimental values are well fitted by those from a calculation of HeNRY due to the dipole velocity approximation. A Rydberg series converging to the ${ }^{5} \mathrm{~S}$-state of the ion is clearly identified from the ionization measurements and is shown to ionize.
\end{abstract}

In neuerer Zeit sind in mehreren Veröffentlichungen Berechnungen des Photoionisierungsquerschnittes von atomarem Sauerstoff und atomarem Stickstoff dargestellt worden. Sauerstoff und Stickstoff sind beide in atomarer Form instabil. Absorptionsuntersuchungen an diesen Systemen wurden aus diesem Grunde überwiegend an der ,,aktiven“ Form, d. h. an dem Teilchengemisch, das eine elektrische Ladung verläßt, vorgenommen. Diese Gemische bestehen in der Hauptsache aus den jeweiligen Atomen und nichtdissoziierten Molekülen, die teilweise angeregt sind. Eine Messung von Ionisierungsquerschnitten muß aber zweckmäßigerweise an den reinen Komponenten erfolgen. Dafür sind Teilchen-

1 F. J. Comes, A. Elzer u. F. Speier, Z. Naturforsch. 23 a, 114 [1968]. strahlen erforderlich, die die jeweiligen Atome in definierten Atomzuständen enthalten. Solche Experimente sind zuerst an Wasserstoff- und Sauerstoffatomstrahlen durchgeführt worden ${ }^{1,2}$. In dieser Arbeit soll über die Photoionisation des Stickstoffatoms im Grundzustand berichtet werden.

\section{Aufbau der Apparatur}

Die experimentelle Anordnung zur Messung der Photoionisation an Atomstrahlen ist schon an anderer Stelle beschrieben worden ${ }^{1}$. Die Meßapparatur bestand aus einem Monochromator in Seya-Anordnung und einem Flugzeitmassenspektrometer. Atom- und Photonenstrahl kreuzen sich senkrecht im Ionisationsraum des Massenspektro-

2 F. J. Comes, F. Speier u. A. Elzer, Z. Naturforsch. 23 a, 125 [1968]. 
meters. Senkrecht zu beiden werden die gebildeten Ionen ins Ionenflugrohr eingeschossen. Im Gegensatz zu früheren Messungen an Wasserstoff- und Sauerstoffatomstrahlen 1,2 wurden die Stickstoffatome in einer kondensierten Entladung erzeugt. In nicht kondensierten Entladungen wird nur eine geringe Stickstoffatomkonzentration erhalten.

Das Entladungsrohr hatte eine Länge von etwa $130 \mathrm{~cm}$ und war $\mathrm{u}$-förmig angeordnet. Bei einem Druck von 0,1 bis 0,2 Torr brannte zwischen zwei Aluminiumelektroden eine durch $0,2 \mu \mathrm{F}$ kondensierte Entladung in Stickstoff, dem 10\% Argon zugesetzt waren. Argon wurde zur Temperaturmessung des aus dem Spalt der Atomstrahlanordnung ausströmenden Gases benutzt ${ }^{2}$. Das Edelgas hatte keinen meßbaren Einfluß auf die Entladung. Die Entladung zündete zweimal pro Sekunde bei einer Spannung von $3,5 \mathrm{kV}$. Bei dieser Frequenz war die Stickstoffatomproduktion hinsichtlich Stabilität und Intensität optimal. Wegen der langen Lebensdauer der Stickstoffatome wirkt sich bei der eingestellten Meßgeschwindigkite diese kleine Entladungsfrequenz nicht aus. Die $\mathrm{N}^{+}$-Ionenstromanzeige war stabil. Die erreichte Atomkonzentration lag zwischen 25 und $30 \%$. Es konnten auch Atomkonzentrationen bis zu 50\% erzielt werden. Die Messungen wurden jedoch aus Stabilitätsgründen bei einer Atomkonzentration von 25\% durchgeführt.

\section{Meßergebnisse und Diskussion}

Die Messung des N-Ionisierungsquerschnittes wurde in zwei Schritten durchgeführt: 1. Messung der N-Ionisierung als Funktion der eingestrahlten Wellenlänge in willkürlichen Einheiten und 2. Bestimmung des Querschnittes des N-Atoms für eine feste Wellenlänge $\lambda_{0}$ in absoluten Einheiten. Teil 1 der Messung bestand also darin, die $\mathrm{N}^{+}$-Produktion nach Gl. (1):

$$
\mathrm{N}\left({ }^{4} \mathrm{~S}\right)+h v \rightarrow \mathrm{N}^{+}+\mathrm{e}^{-}
$$

als Funktion der Wellenlänge zu bestimmen und auf die Photoelektronenausbeute einer Goldkatho$\mathrm{de}^{1} \mathrm{zu}$ beziehen. Dabei sind alle diejenigen Prozesse auszuscheiden, die $\mathrm{zu}$ einer $\mathrm{N}^{+}$-Bildung durch andere Vorgänge führen. Einmal führt die Photoionisation von metastabil angeregten $\mathrm{N}$-Atomen schon vor der Ionisierungsgrenze zur $\mathrm{N}^{+}$-Bildung, zum anderen wird durch dissoziative Ionisation aus $\mathrm{N}_{2}$ dieses Ion gebildet. Zur s $\mathrm{s}^{2} \mathrm{p}^{3}$-Elektronenkonfiguration des $\mathrm{N}$-Atoms gehören neben dem ${ }^{4} \mathrm{~S}$-Grundzustand die beiden angeregten Zustände ${ }^{2} \mathrm{D}$ und ${ }^{2} \mathrm{~S}$. Beide Zustände sind metastabil. Da ein Übergang in den Ionengrundzustand optisch erlaubt ist, liegen die Ionisierungsgrenzen oberhalb $852 \AA$ (1020 bzw. $1130 \AA$ ). Der Beitrag metastabiler Atome zur $\mathrm{N}^{+}$. Ionisierung kann also durch Messung der $\mathrm{N}^{+}$-Bildung unmittelbar vor der Ionisierungsgrenze des

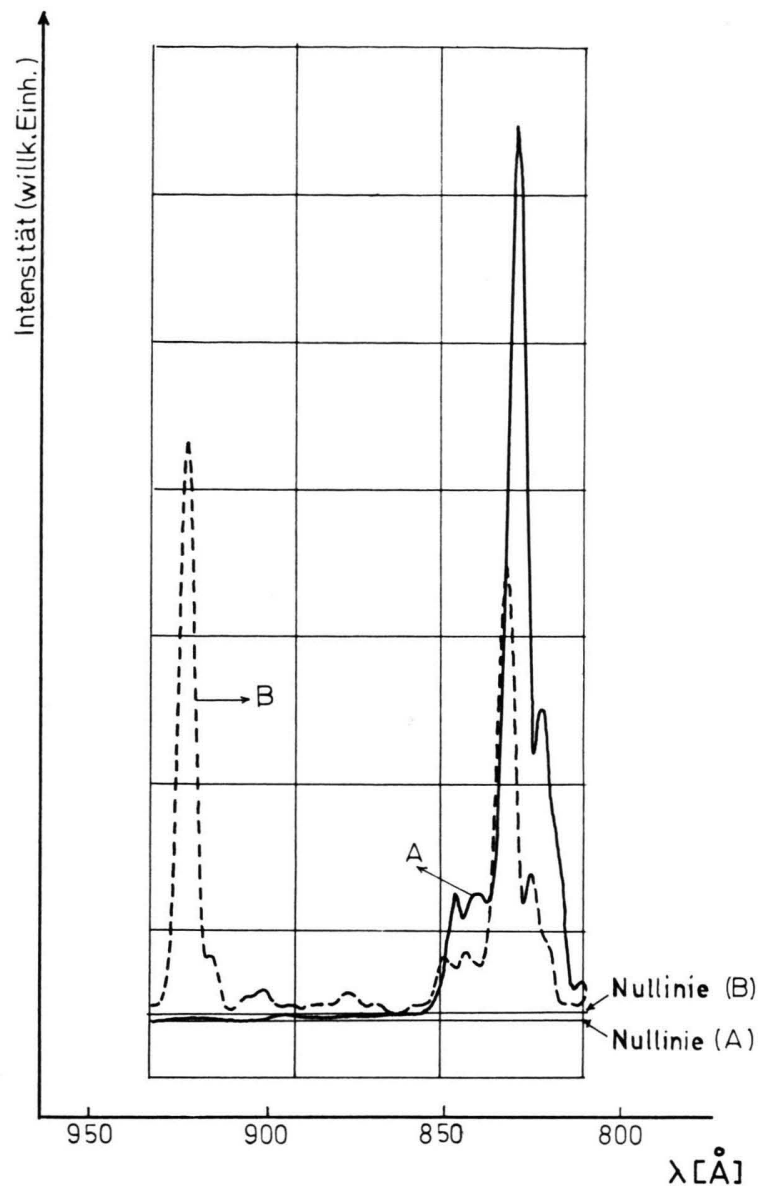

Abb. 1. $\mathrm{N}^{+}$-Ionenstrom (A) und Photoelektronenstrom (B) als Funktion der Wellenlänge.

${ }^{4}$ S-Atoms geprüft werden. Abb. 1 zeigt den $\mathrm{N}^{+}$Ionenstrom für die Wellenlängen $830 \AA$ bis $923 \AA$. Bei $852 \AA$ setzt die $\mathrm{N}^{+}$-Bildung scharf ein. Der Anteil metastabiler $\mathrm{N}$-Atome am gemessenen $\mathrm{N}^{+}$. Ionenstrom ist maximal $0,3 \%$. Die Bildung von Stickstoffionen durch dissoziative Ionisation nach Gl. (2) setzt erst bei der Schwellenenergie $24,4 \mathrm{eV}$ (508 $\AA$ ) ein.

$$
\mathrm{N}_{2}+h v \rightarrow \mathrm{N}^{+}+\mathrm{N}+\mathrm{e}^{-} .
$$

Bei einer früheren Messung war der Wert $511 \AA$ gefunden worden ${ }^{3}$. Der Beitrag der Ionenbildung nach (2) war meßbar und wurde in der Berechnung des Wirkungsquerschnittes berücksichtigt.

Für den zweiten Teil der Messung wurde das Massenspektrometer zunächst durch eine Ionisationskammer ersetzt und die $\mathrm{N}_{2}^{+}$-Bildung für die feste Wellenlänge $\lambda_{0}=554 \AA$ bestimmt. Der Wir- 
kungsquerschnitt $\sigma\left(\mathrm{N}_{2}\right)$ für den Prozeß

$$
\mathrm{N}_{2}+h v \rightarrow \mathrm{N}_{2}^{+}+\mathrm{e}^{-}
$$

kann aus den Meßwerten für den $\mathrm{N}_{2}$-Druck, den Ionenstrom und den Photozellenstrom gewonnen werden. Die Messungen wurden für 10 verschiedene $\mathrm{N}_{2}$-Drucke durchgeführt. Die Wellenlänge $554 \AA$ reicht nicht für die dissoziative Ionisation nach (2) aus und liegt in einem Bereich der $\mathrm{N}_{2}$-Ionisierungskurve, in dem keine oder nur geringe Struktur zu erwarten ist ${ }^{3,4}$. Die Berechnung des $\mathrm{N}_{2}$-Ionisierungsquerschnittes $\sigma(\mathrm{N})$ kann nach Gl. (4)

$$
\sigma(\mathrm{N})=\sigma\left(\mathrm{N}_{2}\right) \frac{J\left(\mathrm{~N}^{+}\right)}{J\left(\mathrm{~N}_{2}^{+}\right)} \cdot \frac{A\left(\mathrm{~N}_{2}^{+}\right)}{A\left(\mathrm{~N}^{+}\right)} \cdot \frac{1-\alpha}{\alpha}
$$

erfolgen. Dazu müssen für eine bestimmte Atomkonzentration $\alpha$ das Ionenstromverhältnis

$$
J\left(\mathrm{~N}^{+}\right) / J\left(\mathrm{~N}_{2}^{+}\right)
$$

und das Verhältnis der Sekundärelektronenausbeute $A\left(\mathrm{~N}_{2}^{+}\right) / A\left(\mathrm{~N}^{+}\right)$mit dem Massenspektrometer bestimmt werden. Nach Berücksichtigung der Temperatur des Probengases im Atomstrahl durch eine Argonmessung ${ }^{2}$ wurde die Atomkonzentration am $\mathrm{N}_{2}^{+}$-Strom mit und ohne Entladung bestimmt. Das Verhältnis der Sekundärelektronenausbeuten ergibt sich aus der mittleren Impulshöhe der von den beiden Ionensorten am Ausgang des Multipliers hervorgerufenen Poisson-Verteilungen. Zur Bestimmung der Atomkonzentration muß noch der Anteil metastabiler Moleküle im Strahl bestimmt werden. Abb. 2 zeigt den $\mathrm{N}_{2}^{+}$-Strom im Bereich zwischen $765 \AA$ und $851 \AA$. Vor der Ionisierungsgrenze $796 \AA$ werden kleine $\mathrm{N}_{2}^{+}$-Ströme gemessen, deren Anteil verglichen mit der Ionisierung unterhalb der Grenzwellenlänge maximal $1,5 \%$ des Ionenstroms ausmacht.

Der Wirkungsquerschnitt $\sigma(\mathrm{N})$ für die Photoionisation des Stickstoffatoms im Grundzustand für den gesamten Wellenlängenbereich wird durch Anpassen der Relativwerte an den für $554 \AA$ gemessenen Absolutwert erhalten. In Abb. 3 sind die Meßpunkte und eine diese Punkte verbindende mittlere Kurve $\mathrm{M}$ angegeben. Außerdem enthält die Abbildung weitere Kurven A, B und C, die den von HENRY ${ }^{5}$ gerechneten Wirkungsquerschnitt in der Dipollängen- (A) und Dipolgeschwindigkeitsnähe-

3 F. J. Comes u. W. Lessmann, Z. Naturforsch. 19a, 65 [1964].

4 R. E. Huffman, Y. Tanaka u. J. C. Larrabee, J. Chem. Phys. 39, 910 [1963].

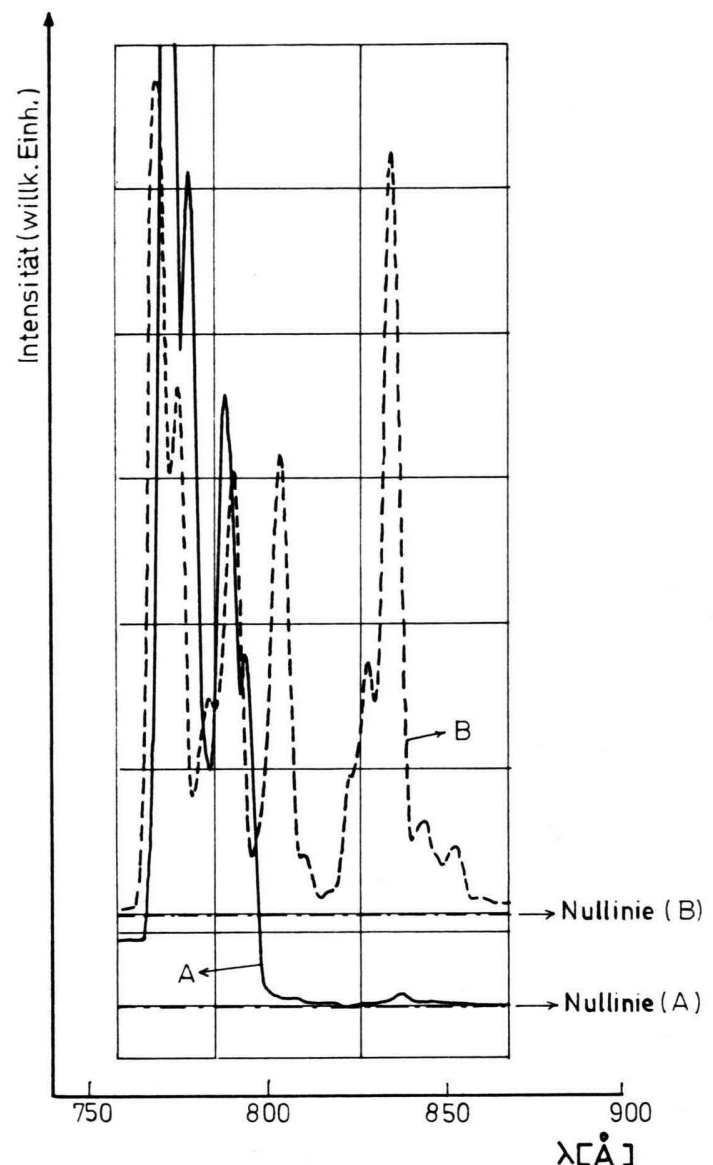

Abb. 2. $\mathrm{N}_{2}{ }^{+}$-Ionenstrom (A) und Photoelektronenstrom (B) als Funktion der Wellenlänge.

rung (B) sowie den von Dalgarno und Parkinson 6 gerechneten Wirkungsquerschnitt in der Dipolgeschwindigkeitsnäherung (C) darstellen. Die HeNRYschen Werte nach der Dipolgeschwindigkeitsnäherung werden bis zu einer Wellenlänge von $500 \AA \mathrm{am}$ besten durch die Meßwerte wiedergegeben. Unter $500 \AA$ weichen jedoch die berechneten Werte dieser Näherung stark von den Meßwerten ab. Wie aus Abb. 3 zu sehen ist, hat die Meßkurve eine wesentlich stärkere Neigung als die der berechneten, deren Neigung im kurzwelligen Teil des gezeigten Wellenlängenbereiches etwa gleich sind. Eine charakteristische Abweichung der Meßkurve tritt zwischen 600 und $700 \AA$ auf. In Absorptions- und Ionisations-

5 R. J. W. Henry, J. Chem. Phys. 45, 4357 [1966].

6 A. Dalgarno u. D. Parkinson, J. Atmospheric Terrest. Phys. 18, 335 [1960]. 


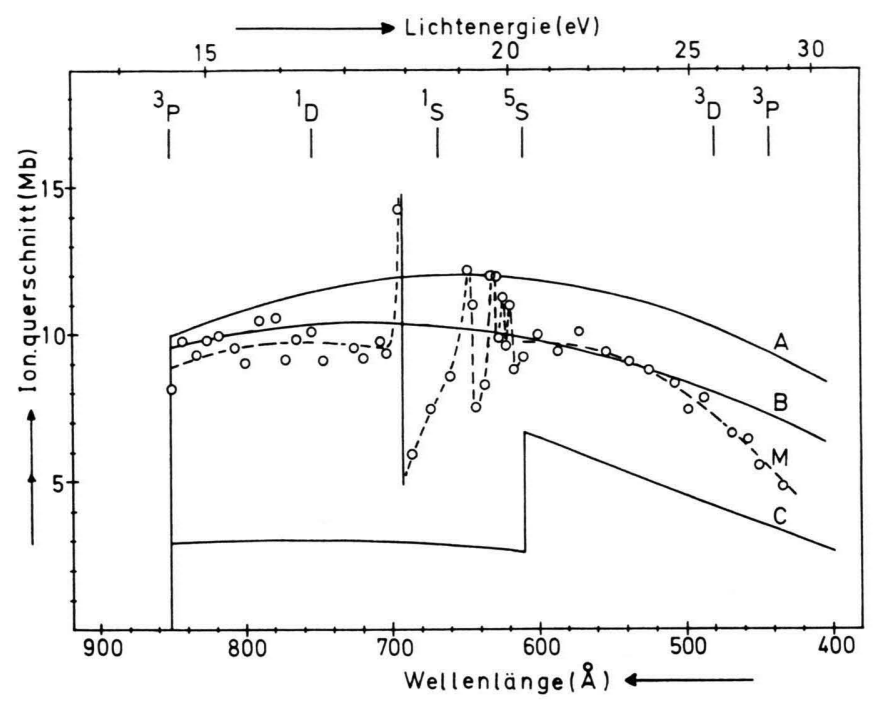

Abb. 3. Wirkungsquerschnitt für die Photoionisation des N-Atoms. M: gemessene Kurve; A u. B: nach Henry 11 (Dipollängen- und Dipolgeschwindigkeitsnäherung); C: nach Dalgarno u. PARKIN. son $^{6}$ (Dipolgeschwindigkeitsnäherung). messungen 7,8 konnte gezeigt werden, daß in diesem Spektralbereich eine Rydberg-Serie existiert, die zum ${ }_{5}^{5}$-Zustand des Ions konvergiert und deren Terme durch Autoionisation zerfallen. Der ${ }^{5} \mathrm{~S}-Z$ Zustand ist der niedrigste Zustand des Ions, in den ein optisch erlaubter Übergang aus dem Grundzustand des NAtoms erwartet werden kann. Die aus Absorptionsmessungen in aktivem Stickstoff ${ }^{7}$ gefundene Konvergenzgrenze stimmt gut mit dem von ERIKSson 9 festgelegten Wert für die Anregungsenergie dieses Zustandes überein. Die Rydberg-Serie wird durch die Übergänge ${ }^{4} \mathrm{~S}^{0}-2 \mathrm{~s} 2 \mathrm{p}^{3}\left({ }^{5} \mathrm{~S}^{0}\right) n \mathrm{p}^{4} \mathrm{P}$ gekennzeichnet ${ }^{10}$. Die Auswahlregeln lassen eine Wechselwirkung dieser Zustände mit den kontinuierlichen $\mathrm{Zu}$ ständen der Serien $2 \mathrm{p}^{2}\left({ }^{3} \mathrm{P}\right) n \mathrm{~s}$ und $2 \mathrm{p}^{2}\left({ }^{3} \mathrm{P}\right) n \mathrm{~d} \mathrm{zu}$. Die durch die Autoionisation hervorgerufene Verbreiterung und Asymmetrie der Absorptionslinien ist deutlich zu erkennen. Daraus war ein Wert $Q \approx 1$ für den Parameter der Linienform gefolgert worden ${ }^{8}$. Die durch die autoionisierende RydbergSerie hervorgerufene Abweichung von der theoretischen Ionisierungskurve folgt daraus, daß diese Übergänge bei der Berechnung gar nicht berück-

7 P. K. Carroll, R. E. Huffman, J. C. Larrabee u. Y. TANAKA, Astrophys. J. 146, 553 [1966].

8 F. J. Comes u. A. Elzer, Phys. Letters 25 A, 334 [1967].

9 K. B. S. Eriksson, Ark. Fys. 13, 303 [1958]. sichtigt wurden. Dieses soll in jetzt laufenden Rechnungen vorgenommen werden ${ }^{11}$.

Die Meßpunkte zeigen in der Nachbarschaft der Ionisierungsgrenze eine starke Streuung. Aus der Messung geht eindeutig hervor, daß die stärkeren Abweichungen außerhalb der Fehlergrenze liegen und nicht durch statistische Streuung erklärt werden können. Übergänge in die Ionenzustände ${ }^{1} \mathrm{D}$ und ${ }^{1} \mathrm{~S}$, die sich ebenfalls aus der Konfiguration $2 \mathrm{~s}^{2} 2 \mathrm{p}^{2}$ ergeben und deren Auftrittspotentiale bei $755 \AA$ und $667 \AA$ liegen, sind spinverboten und sollten im Absorptionsspektrum nicht vorkommen.

$\mathrm{Da}$ die Ionisierungskurve nur für die diskreten Wellenlängen und nicht mit einem kontinuierlichen Spektrum vermessen wurde, ist eine Aussage über mögliche neue Terme im Ionisationskontinuum des Stickstoffatoms äußerst schwierig. Es erscheint jedoch möglich, die hier geschilderten Messungen mit dem Hintergrund eines Kontinuums zu wiederholen.

Wir danken der Deutschen Forschungsgemeinschaft für ihre großzügige Unterstützung der Arbeit.

\footnotetext{
$10{ }^{\circ}$ an den Termsymbolen soll die Parität ungerade bezeichnen.

11 R. J. W. Henry, priv. Mitteilung.
} 